\title{
From basic personality to motivation: Relating the HEXACO factors to achievement goals
}

\author{
Felix C. Dinger ${ }^{\text {a,* }}$, Oliver Dickhäuser ${ }^{a}$, Benjamin E. Hilbig ${ }^{b}$, Elena Müller ${ }^{\text {a }}$, \\ Ricarda Steinmayr ${ }^{c}$, Linda Wirthwein ${ }^{c}$ \\ a University of Mannheim, School of Social Sciences, Department of Psychology, DE-68131 Mannheim, Germany \\ b University of Koblenz-Landau, Cognitive Psychology Lab, Department of Psychology, DE-76829 Landau, Germany \\ c Technical University Dortmund, Department of Psychology, Emil-Figge-Straße 50, DE-44227 Dortmund, Germany
}

\section{A R T I C L E I N F O}

\section{Article history:}

Received 16 July 2014

Received in revised form 30 January 2015

Accepted 28 March 2015

Available online xxxx

\section{Keywords:}

Personality

HEXACO

Honesty-Humility

Big Five

Motivation

Achievement goals

\begin{abstract}
A B S T R A C T
Associations between personality and motivation have been investigated using various operationalizations for both constructs, but no study so far has linked the HEXACO personality space to achievement goals. The aim of the present study was thus to explore relations between the HEXACO personality domains and achievement goals focusing particularly on Honesty-Humility. Participants were 173 high-school students with a mean age of $14.49(S D=1.04$ ) years. Data were collected using questionnaires assessing the HEXACO personality inventory and students' mastery goals, performance-approach goals, and performance-avoidance goals for school in general. With few exceptions, findings were consistent with associations reported for the Big Five dimensions and achievement goals. Moreover, Honesty-Humility showed a distinct pattern of systematic relations with achievement goals, correlating positively with mastery goals and negatively with both performance-approach goals and performance-avoidance goals. Finally, relative weights analyses revealed that Honesty-Humility accounted for substantial proportions of explained variance in all achievement goals.
\end{abstract}

(c) 2015 Elsevier Inc. All rights reserved.

\section{Introduction}

What are the relations between personality and motivation? There is ample research addressing this question in general and several studies doing so with regard to one of the most prominent constructs in motivation research - achievement goals - in particular. Yet, most of this literature draws on the classical five-factor model of personality, or the Big Five (e.g., McCrae \& John, 1992). Although this model is still most widely accepted, recent work on personality structure has hinted that, across languages and cultures, a slight variation of some of the basic personality factors and addition of a sixth is actually more appropriate. These extensions of the five-factor approach are subsumed in the HEXACO model of personality that has been used increasingly in recent research - primarily because the new sixth factor, Honesty-Humility, has proved to be predictive regarding various criteria (for recent

\footnotetext{
* Corresponding author. Tel.: +49621 181 2287; fax: +49621 1812206.

E-mail addresses: felix.dinger@uni-mannheim.de (F.C. Dinger), oliver.dickhaeuser@uni-mannheim.de (O. Dickhäuser),hilbig@uni-landau.de (B.E. Hilbig), elemuell@students.mail.uni-mannheim.de (E. Müller), ricarda.steinmayr@tu-dortmund.de (R. Steinmayr), linda.wirthwein@tu-dortmund.de (L. Wirthwein).
}

overviews, see Ashton, Lee, \& De Vries, 2014; Hilbig, Glöckner, \& Zettler, 2014a). However, despite this growing interest in and evidence for the HEXACO model, educational researchers have only recently begun to examine corresponding personality dimensions in relation to learning and achievement in general. Indeed, the HEXACO model has been neglected altogether by scholars investigating individuals' motivation to learn and achieve in particular. The study reported in the following aims to fill this research gap.

\section{Theoretical background}

Decades of research corroborate the major dimensions of individuals' personality to be rather stable constructs with substantial heritability (cf. Bouchard, 2004; Eysenck, 1990; Funder, 2001). Specifically, scholars conceive the major dimensions of personality as source traits that may be used to explain individual differences in more specific traits or habits. In line with this reasoning, we construe the HEXACO personality domains as predictors of students' achievement goals. Before we delineate our hypotheses about these predictive relations, we will briefly introduce the HEXACO model, sketch the achievement goal approach to motivation, and review the literature reporting relations between the Big Five and achievement goals in the following. 


\subsection{The HEXACO model of personality}

Based on the results of lexical studies in various languages and cultures (Ashton \& Lee, 2010; Ashton et al., 2004; Lee \& Ashton, 2008), the HEXACO model subsumes the many ways in which individuals differ in six basic factors: Honesty-Humility $(H H)$, Emotionality (EM), Extraversion (EX), Agreeableness ( $A G)$, Conscientiousness (CO), and Openness to Experience $(O P)$, thus the acronym HEXACO. As hinted above, the HEXACO model varies and extends the Big Five: three of the HEXACO factors $(E X, C O$, and $O P$ ) are essentially equivalent to the corresponding Big Five factors. In addition, the HEXACO factors EM and $A G$ are variants of Big Five Neuroticism and Agreeableness. In the HEXACO, EM refers almost exclusively to intra-personal, emotional content (though including emotions of empathy and attachtment to others), whereas inter-personal, social aspects are all subsumed in $A G$ - unlike in the classical Neuroticism and Agreeableness factors which both subsume emotional and social aspects. Most strikingly, however, the HEXACO includes a sixth basic factor, named HonestyHumility (Ashton \& Lee, 2007; Ashton et al., 2014).

Honesty-Humility covers attributes such as being sincere, fairminded, unassuming, and modest versus sly, greedy, boastful, and hypocritical (Lee \& Ashton, 2008, 2012). It thus reflects individual differences in "the tendency to be fair and genuine in dealing with others" (Ashton \& Lee, 2007, p. 156) in the sense of actively cooperating with others (Ackermann, Fleiß, \& Murphy, in press; Hilbig, Zettler, Leist, \& Heydasch, 2013) and subsumes the facets Sincerity, Fairness, Greed Avoidance, and Modesty in the HEXACO Personality Inventory-Revised (Lee \& Ashton, 2004, 2006).

\subsection{The achievement goal approach to motivation}

Achievement goal theory seeks to explain and predict the direction and intensity of individuals' behavior in achievement situations from a cognitive viewpoint (Dweck \& Leggett, 1988; Elliot, 1999). More precisely, achievement goal theory posits that individuals may engage in the same activities for different reasons, or in other words, that individuals may pursue different achievement goals. These achievement goals in turn are hypothesized to have an effect on individuals' affect, cognition, and behavior in achievement situations. In the present research we draw on one of the most frequently investigated achievement goal models, i.e. the trichotomous framework (Elliot, 1997; Elliot \& Church, 1997; Elliot \& Harackiewicz, 1996) that distinguishes between three different achievement goals: mastery goals denote aiming for developing one's competence and accomplishing task mastery, performance-approach goals denote aiming for demonstrating one's high competence by outperforming others, and performance-avoidance goals denote aiming for avoiding the demonstration of one's incompetence by not doing worse than others.

\subsection{Relations between personality and achievement goals}

As hinted above, research investigating the relations between personality and achievement goals has mainly focused on the Big Five traits (e.g., McCrae \& John, 1992) with its dimensions Neuroticism ( $N$ ), Extraversion $(E)$, Openness to Experience $(O)$, Agreeableness $(A)$, and Conscientiousness $(C)$. Elliot and Thrash (2002) developed a theoretical model regarding the associations between personality and achievement goals. They assumed the existence of approach and avoidance personality dimensions, with $E$ belonging to the approach component and $N$ belonging to the avoidance component. Personality approach components (such as $E$ ) should be positively associated with mastery goals and performance-approach goals, whereas personality avoidance components (such as $N$ ) should be positively associated with performanceavoidance goals. Furthermore, the authors hypothesized that $N$ is additionally linked to normative goals such as performance-approach goals. Elliot and Thrash (2002) found support for these hypotheses across several studies although the correlations between the personality dimensions and achievement goals were rather small. Unfortunately, Elliot and Thrash (2002) did not postulate theoretical assumptions for the remaining Big Five dimensions and their associations with achievement goals.

Focusing on adults in educational and occupational settings, Payne, Youngcourt, and Beaubien (2007) conducted a meta-analysis on the associations between several variables, inter alia the Big Five, and achievement goals. Including up to 16 studies, the authors corroborated that $E$ was positively related to mastery goals $(r=.24)$. Furthermore, $O$ $(r=.34), A(r=.15)$, and $C(r=.26)$ were also positively associated with mastery goals, whereas $N$ was negatively related to mastery goals $(r=-.14)$. Again in line with predictions of Elliot and Thrash (2002), $N$ was positively related to performance-avoidance goals $(r=$ .29). Additionally, $E(r=-.24), O(r=-.19), A(r=-.15)$, and $C$ $(r=-.14)$ were found to be negatively associated with performanceavoidance goals. Findings were less conclusive regarding the associations between the Big Five and performance-approach goals because Payne et al. (2007) merged the latter into one category with global performance goals (i.e. performance goals not differentiated into performance-approach goals and performance-avoidance goals). The respective results, thus, do not allow a clear interpretation.

Other studies investigating samples of college or university students have also partly confirmed the assumptions by Elliot and Thrash (2002) regarding the relation of $E$ to mastery goals and of $N$ to performance-avoidance goals (e.g., Bipp, Steinmayr, \& Spinath, 2008; Day, Radosevich, \& Chasteen, 2003; Zweig \& Webster, 2004). However, in contrast to the findings reported by Elliot and Thrash (2002), the aforementioned studies only observed negligible associations between $E$ and performance-approach goals.

These and other studies also reported results for the associations between the remaining Big Five dimensions and achievement goals (e.g., Bipp et al., 2008; Day et al., 2003; Vermetten, Lodewijks, \& Vermunt, 2001; Zweig \& Webster, 2004): 0 was positively related to mastery goals $(.16 \leq r \leq .38)$. Slightly negative or negligible correlations $(-.21 \leq r \leq .00)$ were reported for $O$ and performance-avoidance goals (ibid.). Furthermore, mainly positive relations between $A$ and mastery goals $(.04 \leq r \leq .20)$ as well as slightly negative associations between $A$ and performance-avoidance goals $(-.13 \leq r \leq-.02)$ were reported (ibid.). The same pattern of associations was found for the relationship between $C$ and mastery goals $(.09 \leq r \leq .26)$ and $C$ and performanceavoidance goals $(-.22 \leq r \leq .07$; ibid.). The correlations between $O, A$, and $C$ and performance-approach goals were small or negligible $(r \leq .15$; ibid.).

However, there are also contrary findings: Steinmayr, Bipp, and Spinath (2011), examining a high-school student sample, found $N$ and $E$ to be nearly uncorrelated with mastery goals, whereas $A$ and $C$ were positively correlated with performance-approach goals. The other results were in line with the research on adults. Wang and Erdheim (2007), analyzing a working sample, found that $E$ was only associated with mastery goals, whereas $N$ was positively correlated with both performance-approach goals and performance-avoidance goals - again, confirming the assumptions by Elliot and Thrash (2002).

The studies reviewed so far almost exclusively report relations between Big Five dimensions and achievement goals. However, at least one investigation went into further detail: Bipp et al. (2008) also examined associations of personality facets with achievement goals. Their evidence may be especially informative for the present research because the respective findings could provide first hints on how $\mathrm{HH}$ might be related to achievement goals. For instance, Modesty (a facet scale of $A$ within the Big Five model, but belonging to $\mathrm{HH}$ within the HEXACO framework) was found to be negatively associated with performanceapproach goals $(r=-.26)$.

\section{The present study and hypotheses}

The present research aimed at gathering first evidence regarding the relations between students' personality in terms of the HEXACO model 


\section{دريافت فورى ـ ـ متن كامل مقاله}

\section{ISIArticles}

مرجع مقالات تخصصى ايران

ل امكان دانلود نسخه تمام متن مقالات انكليسى ل امكان دانلود نسخه ترجمه شده مقالات ل يذيرش سفارش ترجمه تخصصى $\checkmark$ ل امكان جستجو در آرشيو جامعى از صدها موضوع و هزاران مقاله ل امكان دانلود رايكان r صفحه اول هر مقاله

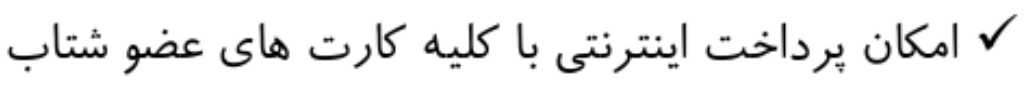
ل دانلود فورى مقاله پِ از برداخت آنلاين

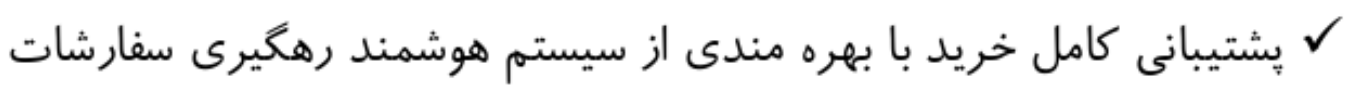

